\title{
Influence of hydrogen-rich saline on hepatocyte autophagy during laparoscopic liver ischaemia-reperfusion combined resection injury in miniature pigs
}

\author{
Ge Bai, Hui Li, Yansong Ge, Qianzhen Zhang, Jiantao Zhang, \\ Mingzi Chen, Tao Liu, Hongbin Wang \\ Heilongjiang Key Laboratory for Laboratory Animals and Comparative Medicine, \\ College of Veterinary Medicine, Northeast Agricultural University, Harbin 150030, China \\ hbwang1940@163.com
}

Received: March 22, $2018 \quad$ Accepted: August 22, 2018

\begin{abstract}
Introduction: The purpose of this study was to investigate the protective effect of hydrogen-rich saline (HRS) against liver ischaemia-reperfusion combined resection injury. Material and Methods: Eighteen miniature pigs were randomly divided into three groups: a sham operated group (sham group, laparoscopic liver ischaemia-reperfusion combined resection injury group (IRI group), and a hydrogen-rich saline intervention group (IRI + HRS group). Samples of hepatic tissue and serum were collected at the time of reperfusion and then $3 \mathrm{~h}, 1 \mathrm{~d}$, and $3 \mathrm{~d}$ post reperfusion. Liver function, oxidative stress, autophagy-related mRNA genes, and protein expression were evaluated. Changes in cell and tissue ultrastructure were examined by transmission electron microscopy. Results: Compared with the sham group, the level of autophagy of hepatocytes increased in the IRI and IRI + HRS groups, corresponding to high oxidative stress and severe liver function injury. Liver function, antioxidant content, autophagy levels, and liver injury were improved after intervention with HRS in the IRI + HRS group compared with the IRI group. Conclusion: Intervention with hydrogen-rich saline could exert a protective effect against liver ischaemia-reperfusion combined resection injury through the reduction of oxidative stress and hepatocyte autophagy.
\end{abstract}

Keywords: miniature pigs, hydrogen-rich saline, liver, ischaemia-reperfusion combined resection, autophagy.

\section{Introduction}

Recently, because of smaller incisions, rapid postoperative recovery reduced postoperative complications, and a decrease in the return to normal function time, laparoscopy in veterinary medicine has grown in popularity (18). These advantages recommended the technique also for in vivo research necessitating surgery.

The liver is critically dependent upon a rich supply of oxygen since it is highly sensitive to hypoxia (7). During hepatic surgery, such as resection and transplantation, the portal circulation is clamped to prevent the loss of blood. It results in temporary ischaemia of the liver, inducing hypoxia in the tissues (21). The absence of oxygen and nutrients from circulating blood during the ischaemic period creates a condition in which the restoration of circulation, somewhat ironically, can be deleterious as opposed to restoring normal function. Degradation of the liver occurs from oxidative damage and inflammation through oxidative stress. This phenomenon is referred as reperfusion injury. Hepatic ischaemia-reperfusion injury is a commonly encountered obstacle appearing in many clinical situations, such as following liver resection surgery and transplantation, liver injury, and post-shock liver failure (13). Therefore, the prevention of hepatic ischaemia-reperfusion injury is a challenge for veterinary clinical therapy.

The mechanisms of ischaemia-reperfusion injury have implicated functions of oxidative stress, intracellular calcium overload, mitochondria, activation of liver Kupffer cells, and release of cytokines and chemokines (28). All of the above conditions result in the production of oxygen-derived free radicals and the recruitment of neutrophils, which further contribute to 
cellular injury and cell death (necrosis or apoptosis). Following hepatic ischaemia-reperfusion, large amounts of reactive oxygen species (ROS) are produced by the xanthine oxidase system, macrophage system, and mitochondrial respiratory chain (11). Moreover, the release of ROS can damage proteins, nucleic acids, cell membranes, and lipids (30), which can directly damage the hepatocytes. Autophagy has also been reported to be related to hepatic ischaemiareperfusion injury. Hepatocytes in thus injured liver degrade damaged and aged mitochondria, in addition to other surplus or dysfunctional organelles through autophagy. Rautou et al. (24) showed that autophagy has mainly prosurvival activity allowing the cell to cope with nutrient starvation and anoxia, while some studies had apparently contradictory results. It is unclear whether the autophagy response is beneficial or detrimental.

The smallest elemental gas molecule, hydrogen, is able to penetrate into the cytoplasm, mitochondria, and nucleus of cells through simple diffusion across the cellular membrane. Recently, it has been demonstrated that hydrogen can selectively scavenge both hydroxyl radicals and peroxynitrite anions of ROS. Moreover, hydrogen has been shown to exhibit protective effects against several types of oxidative stress-induced organ damage. These protective effects occur through the suppression of oxidative stress and apoptosis, the inhibition of inflammatory cell infiltration, and the regulation of pro-inflammatory cytokine expression, as well as inflammation/apoptosis-related signalling pathways (9).

In the current study, we sought to determine whether hydrogen-rich saline (HRS) can attenuate liver ischaemia-reperfusion combined resection injury through anti-autophagic mechanisms.

\section{Material and Methods}

Model establishment and sample collection. Eighteen healthy 3-5-month-old Bama miniature pigs (half of them male and half female), weighing $30 \pm$ $3.9 \mathrm{~kg}$ were examined physically and haematologically to ensure that all animals were in good health prior to the study's initiation. The pigs were randomly divided into three equal groups as follows: a sham operation group (sham group) in which pigs were subjected to laparoscopic surgery manipulating the liver lobes but without liver ischaemia-reperfusion or partial resection; a laparoscopic liver ischaemia-reperfusion combined resection injury group (IRI group), utilising a 4-portal approach where laparoscopic liver ischaemia and reperfusion as well as a partial resection were performed after a $60 \mathrm{~min}$ ischaemia; and a hydrogenrich saline group (IRI + HRS group) in which the laparoscopic operation was performed the same way as in the IRI group, but included portal vein catheterisation before reperfusion and administration of a portal vein injection of HRS $(10 \mathrm{~mL} / \mathrm{kg}) 10 \mathrm{~min}$ prior to reperfusion.

Hydrogen was dissolved in $0.9 \%$ sterilised saline under $0.4 \mathrm{MPa}$ pressure for $6 \mathrm{~h}$ in order to reach the supersaturated level, using a hydrogen generator (Saikesaisi hydrogen energy, China). Hydrogen-rich saline was freshly prepared every time to ensure that the concentration of the hydrogen was maintained at more than $0.6 \mathrm{mmol} / \mathrm{L}$.

All pigs were administered the same general anaesthesia and were monitored and managed similarly after a $12 \mathrm{~h}$ fasting period in which water was also withheld for the final $2 \mathrm{~h}$ prior to surgery. Each pig was pre-medicated subcutaneously with $0.05 \mathrm{mg} / \mathrm{kg}$ of atropine (Beijing Shuanghe Pharmaceutical, China) and $15 \mathrm{~min}$ later intramuscular injections of xylazine $(1 \mathrm{mg} / \mathrm{kg})$ and ketamine hydrochloride $(10 \mathrm{mg} / \mathrm{kg})$ were given and intravenous administration of propofol $(3.5 \mathrm{mg} / \mathrm{kg}$ ) was used to induce anaesthesia. The animal was intubated with a $6.0-7.0 \mathrm{~mm}$ tracheal tube and maintained with $1.5 \%-3 \%$ isoflurane in oxygen after intubation. Laparoscopic hepatic ischaemia-reperfusion and partial resection were performed after establishing a 4-portal approach. After laparoscopic dissection of the liver duodenal ligament and separation of the left and right hepatic arteries and portal vein, the right side of the liver was ligated with a homozygous tourniquet for 60 min to block blood flow to the right part of the liver. Next, the tourniquet was released to initiate reperfusion. After laparoscopic hepatic ischaemiareperfusion, the left hepatic lobe was ligated in a double row. Next, the liver parenchyma was resected proximal to and following the ligation line with a highfrequency electric knife. Intraoperative bleeding was managed with electrocoagulation, gauze, gelatin sponges, and other standard treatments as appropriate. Finally, the resected liver lobe was removed from the abdominal cavity. Then, the laparoscope was removed after a thorough exploration of the abdomen, and the incision was closed by nodule suture.

All animals were housed and monitored in identical environments. Daily clinical observations of the pigs, including mental state, exercise, feeding, wound healing, and defecation were performed for seven days. After anaesthesia, the animals were placed in a warm room to recover. After $6 \mathrm{~h}$ of recovery, animals were allowed water and after $18 \mathrm{~h}$ feed. After surgery, $5 \mathrm{mg}$ of fentanyl (Duragesic; Janssen Pharmaceutical, China) was applied in a patch, and this was repeated three and six days later. All animals received a prophylactic intramuscular injection of cefazolin sodium $(50 \mathrm{mg} / \mathrm{kg}$; Harbin Pharmaceutical Group, China General Pharmaceutical) twice daily for two days. At postoperative day 7, the pigs were euthanised. Pigs were first anaesthetised with xylazine and ketamine hydrochloride, then intravenously injected with $300 \mathrm{mg}$ of xylocaine. Necropsy was performed to examine the condition of the abdominal cavity and postoperative healing. 
Blood samples were collected before operation (Tp), at reperfusion (T0h), $3 \mathrm{~h}$ post reperfusion (T3h), $1 \mathrm{~d}$ post reperfusion (T1d), and $3 \mathrm{~d}$ post reperfusion (T3d). Liver tissues were taken from the same site of the right lobe of the liver at similar times post eutanasia. One liver tissue sample from each pig was fixed in $2.5 \%$ glutaraldehyde for subsequent TEM analysis. A second liver tissue sample was placed in $4 \%$ paraformaldehyde for immunohistochemical staining analysis. Liver tissue samples were also homogenised in ice-cold saline and centrifuged at $2,500 \mathrm{rpm}$ for $10 \mathrm{~min}$. Supernatants were stored at $-20^{\circ} \mathrm{C}$ for further analysis. The final section was frozen immediately in liquid nitrogen and stored at $-80^{\circ} \mathrm{C}$ for further analysis.

Index measurement and method. Serum alanine aminotranspherase (ALT), aspartate transaminase (AST), alkaline phosphatase (AKP), liver homogenate malondialdehyde (MDA), superoxide dismutase (SOD), glutathione (GSH), and catalase (CAT) were measured using the respective detection kits (Nanjing Jiancheng Bioengineering Institute, China) according to the manufacturer's instructions.

Autophagy-related mRNA gene expressions were measured by real-time PCR with a light cycler 480 system (Roche, Switzerland) according to the manufacturer's instructions. The relative abundance of mRNA was calculated according to the method of $2^{-\Delta \Delta C \mathrm{t}}$. The primers (Table 1) used for amplification by qPCR were synthesised by Sangon Biotech (China). Autophagy-related gene protein expressions were measured by Western blot. The blots were quantified using the Image-J software.

Table 1. Primer sequences

\begin{tabular}{|c|c|}
\hline Gene & Primer sequence $\left(5^{\prime}-3^{\prime}\right)$ \\
\hline \multirow{2}{*}{ Beclin 1} & TCCATTACTTGCCACAGCCC (forward) \\
\hline & CCCGATCAGAGTGAAGCTGT (reverse) \\
\hline \multirow{2}{*}{ mTOR } & ACAAGGACACAGCGACTCAG (forward) \\
\hline & CGCGGAACCAGTGAGGTAAT (reverse) \\
\hline \multirow{2}{*}{ p62 } & TACACGAGACCAGTCAACCTAAC (forward) \\
\hline & AGAAGATGCTTGTGCCGAG (reverse) \\
\hline \multirow{2}{*}{ ATG5 } & GGTTTGAATATGAAGGCACACCA (forward) \\
\hline & TGTGATGTTCCAAGGAAGAGCTG (reverse) \\
\hline \multirow{2}{*}{ ATG12 } & CAGAAACAGCCATCCCAGAG (forward) \\
\hline & GCCTTCAGCAGGATGTCAAT (reverse) \\
\hline \multirow{2}{*}{$\beta$-Actin } & TCTGGCAACCACACCTTCT (forward) \\
\hline & TGATCTGGGTCATCTTCTCAC (reverse) \\
\hline
\end{tabular}

Liver tissues were subjected to immunohistochemical staining for microtubuleassociated protein light chain 3B (LC3B). The ultrastructure of hepatocytes was observed under a transmission electron microscope.

Statistical analysis. All statistical tests were performed using SPSS 22.0 software. One-way ANOVA analysis followed by multiple comparisons between groups after a normality test and the assumption of homogeneity of variances was used. The data were expressed as the mean $\pm \mathrm{SD}$ and $\mathrm{P}<0.05$ was considered to be statistically significant.

\section{Results}

Liver function. ALT, AST, and AkP activity in the IRI and IRI + HRS groups showed a tendency to increase at $\mathrm{T} 0 \mathrm{~h}, \mathrm{~T} 3 \mathrm{~h}$, and $\mathrm{T} 1 \mathrm{~d}$, peaking at $\mathrm{T} 1 \mathrm{~d}$, and then decreasing at T3d. ALT, AST, and AKP activity significantly increased in the IRI and IRI + HRS groups compared with the sham group at T0h, T3h, T1d, and T3d. However, ALT and AST activity significantly decreased at $\mathrm{T} 3 \mathrm{~h}$ and $\mathrm{T} 1 \mathrm{~d}$ and AKP activity significantly decreased at T1d in the IRI + HRS group compared with the IRI group $(\mathrm{P}<0.05$, Figs $1 \mathrm{~A}, 1 \mathrm{~B}$, and $1 \mathrm{C})$.

Oxidant stress. MDA concentration in the IRI and IRI + HRS groups showed a tendency to increase at T0h and T3h, peaking at T3h, and then decreasing at T1d and T3d (Fig. 2A). In comparison to the sham group, MDA levels significantly increased at T0h, T3h, and T1d in the IRI and IRI + HRS groups. At T3h and T1d, the levels significantly decreased in the IRI + HRS group compared with the IRI group $(\mathrm{P}<0.05$, Fig. 2A). The concentrations of CAT, GSH, and SOD in liver homogenates of the IRI and IRI + HRS groups decreased at $\mathrm{T} 0 \mathrm{~h}$ and $\mathrm{T} 3 \mathrm{~h}$ and bottomed out at $\mathrm{T} 3 \mathrm{~h}$ before gradually increasing at T1d and T3d (Figs 2B, $2 \mathrm{C}$, and 2D). The concentrations of CAT, GSH, and SOD significantly decreased at T0h, T3h, and T1d in the IRI and IRI + HRS groups compared with those of the sham group. At $\mathrm{T} 3 \mathrm{~h}$ and $\mathrm{T} 1 \mathrm{~d}$, the levels significantly increased in the IRI + HRS group compared with the IRI group $(\mathrm{P}<0.05$, Figs $2 \mathrm{~B}, 2 \mathrm{C}$, and 2D).

The subcellular ultrastructure of hepatocytes. TEM observation indicated that liver of the sham group (Figs 3A and 3B) appeared normal, whereas the IRI (Figs 3C and 3D) and IRI + HRS (Figs 3E and 3F) groups showed pronounced swelling of organelles (mitochondria, Golgi apparatus, endoplasmic reticulum, etc.), membrane rupture, vacuolisation, and a reduction in the number of ribosomes. Mitochondrial damage in the IRI + HRS group was mild and the number of mitochondria was higher than in the IRI group. 

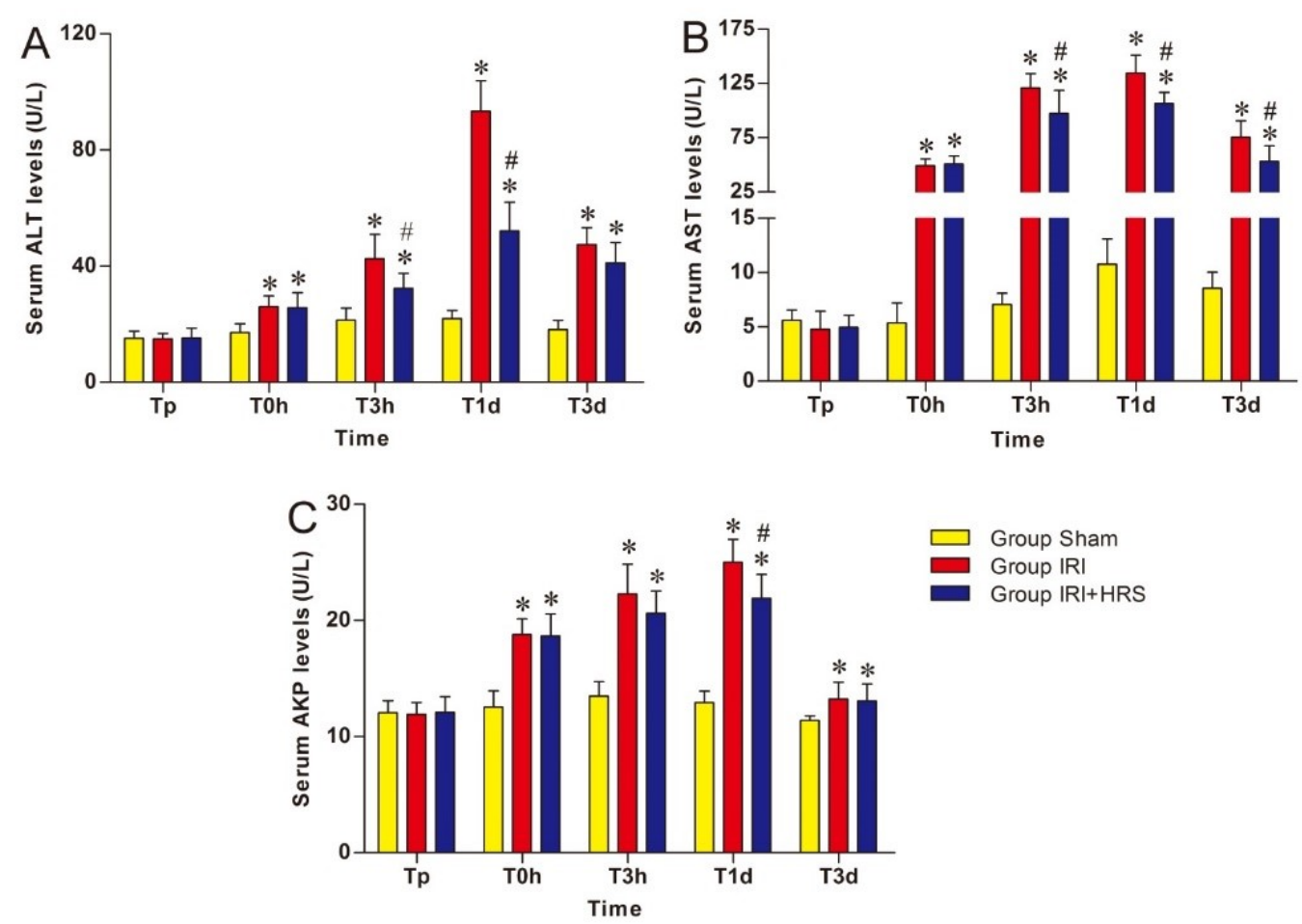

Fig. 1. Levels of ALT (A), AST (B), and AKP (C) in serum. Values are mean \pm standard deviation. *Significant difference from sham group. \# Significant difference of IRI + HRS group from IRI group
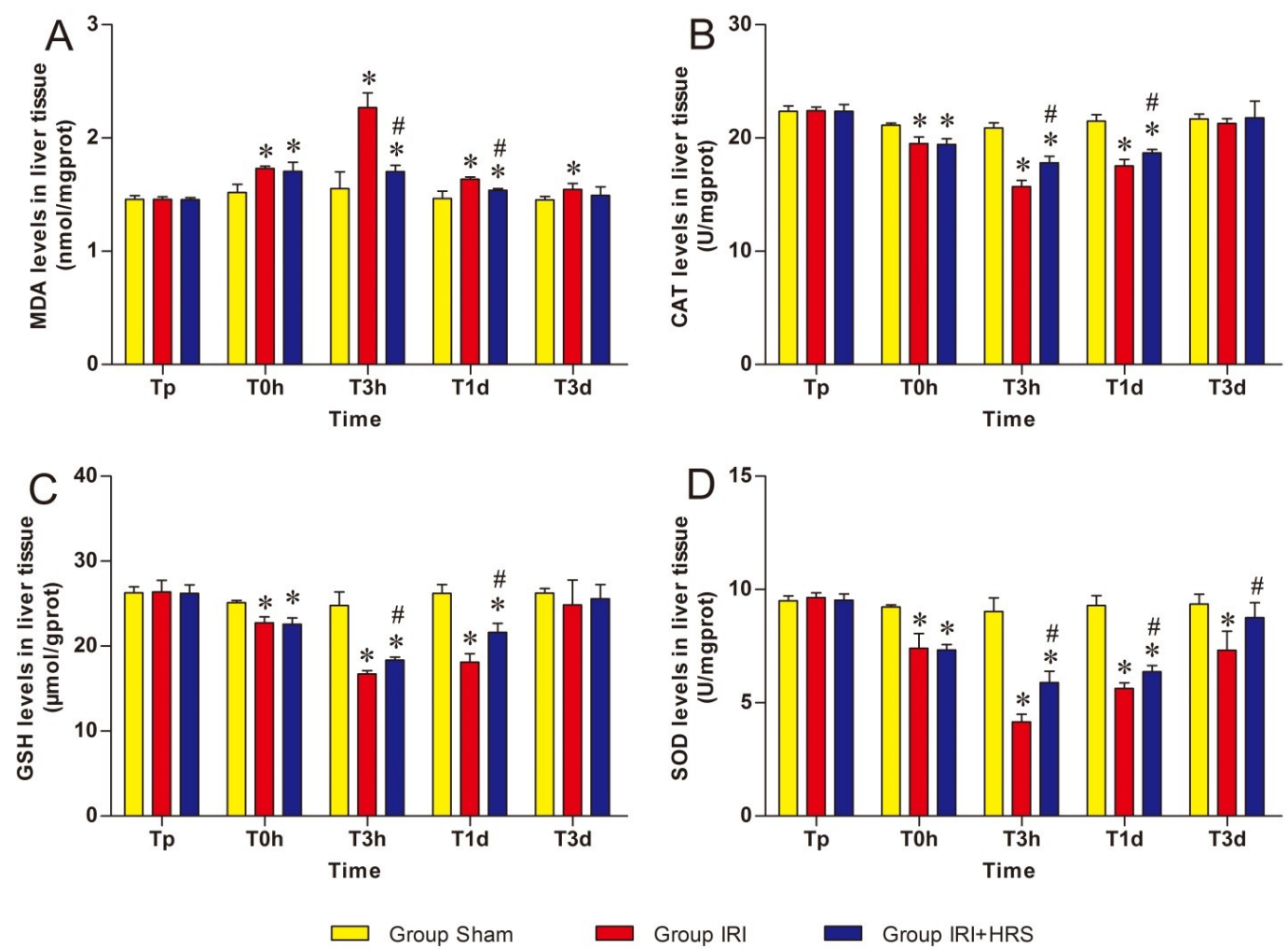

Fig. 2. Levels of MDA (A), CAT (B), GSH (C), and SOD (D) in liver tissue. Values are mean \pm standard deviation. *Significant difference from sham group. \# Significant difference of IRI + HRS group from IRI group 
Autophagy-related mRNA genes and protein expression. Gene expression of the autophagy-related gene Beclin 1, mammalian target of rapamycin (mTOR), sequestosome1 (p62), autophagy-related gene 5 (ATG5), and autophagy-related gene 12 (ATG12) were measured using RT-PCR in liver tissue. The mRNA expression of Beclin 1, ATG5, and ATG12 in the IRI and IRI + HRS groups increased from T0h to $\mathrm{T} 3 \mathrm{~h}$, with a peak at T3h, before gradually decreasing through T1d to T3d (Figs 4A, 4D, and 4E). At T0h, $\mathrm{T} 3 \mathrm{~h}$, and $\mathrm{T} 1 \mathrm{~d}$, the mRNA expression of Beclin 1, ATG5, and ATG12 significantly increased in the IRI and IRI + HRS groups compared with the sham group. At T3h and T1d, the expression of Beclin 1 and ATG12 significantly decreased in the IRI + HRS group compared with the IRI group $(\mathrm{P}<0.05$, Figs $4 \mathrm{~A}$ and $4 \mathrm{E}$ ), and at T3h, the expression of ATG5 significantly decreased in the IRI + HRS group compared with the IRI group $(\mathrm{P}<0.05$, Fig. 4D). In addition, gene expression of p62 and mTOR in the IRI and IRI + HRS groups decreased slightly at $\mathrm{T} 0 \mathrm{~h}$ and $\mathrm{T} 3 \mathrm{~h}$, and bottomed out at $\mathrm{T} 3 \mathrm{~h}$, before gradually increasing at T1d and T3d (Figs 4B and 4C). At T0h, T3h, and T1d, p62 and mTOR gene expression decreased significantly in the IRI and IRI + HRS groups compared with the sham group. However, at T3h and T1d, p62 and mTOR gene expression significantly increased in the IRI + HRS group in comparison to the IRI group $(\mathrm{P}<0.05$, Figs 4B and 4C).

To support gene expression studies, Western blot analysis was used to measure the expression of the autophagy-associated proteins Beclin1, p62, and microtubule-associated protein-1 light chain 3-B (LC3B) in liver tissue homogenates. The expression of Beclin 1 and LC3B in the IRI and IRI + HRS groups exhibited a tendency to increase at T0h and T3h, with peak protein levels being observed at $\mathrm{T} 3 \mathrm{~h}$, and then gradually decreasing at T1d and T3d (Figs 5A and 5B). The expression of p62 in the IRI and IRI + HRS groups tended to decrease at $\mathrm{T} 0 \mathrm{~h}$ and $\mathrm{T} 3 \mathrm{~h}$ and to reach its minimum level at $\mathrm{T} 3 \mathrm{~h}$, then gradually increased at $\mathrm{T} 1 \mathrm{~d}$ and T3d (Fig. 5C).

At T0h, T3h, and T1d, protein expression levels of Beclin 1 and LC3B were significantly higher in the IRI and IRI + HRS groups in relation to the sham group. However, the levels were significantly lower in the IRI + HRS group compared to the IRI group at T3h and T1d ( $\mathrm{P}<0.05$, Figs 5A and 5B). In addition, at T0h and $\mathrm{T} 3 \mathrm{~h}$ the expression of $\mathrm{p} 62$ significantly decreased in the IRI and IRI + HRS groups relative to the sham group, and was significantly increased in the IRI + HRS group relative to the IRI group at $\mathrm{T} 3 \mathrm{~h}(\mathrm{P}<0.05$, Fig. $5 \mathrm{C})$.

Immunohistochemistry. Compared with the sham group (Figs 6A and 6B), apparent positive staining (brown stained tissues) was observed in the IRI group (Figs 6C and 6D) and in the IRI + HRS group (Figs $6 \mathrm{E}$ and $6 \mathrm{~F}$ ) at $\mathrm{T} 3 \mathrm{~h}$, although the frequency of LC3B positive cells was notably decreased in the IRI + HRS group compared to those in the IRI group $(100 \times$ and $400 \times$ magnification).
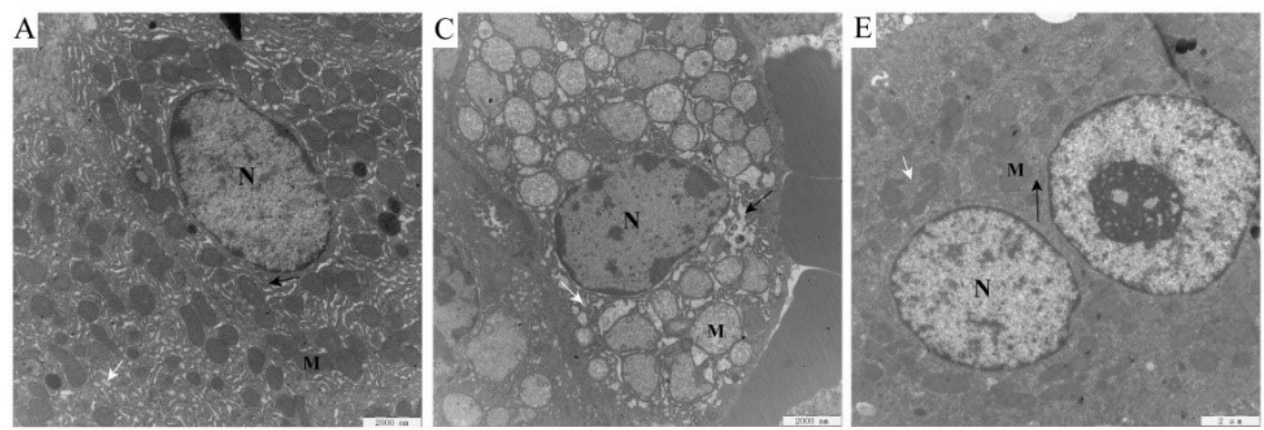

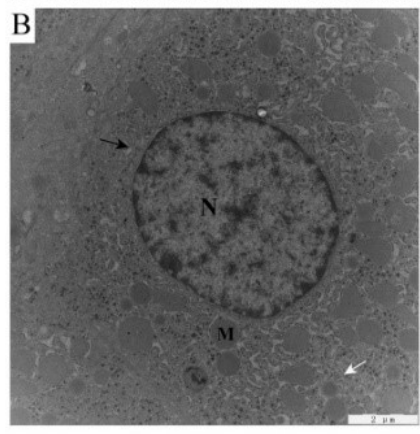

Sham

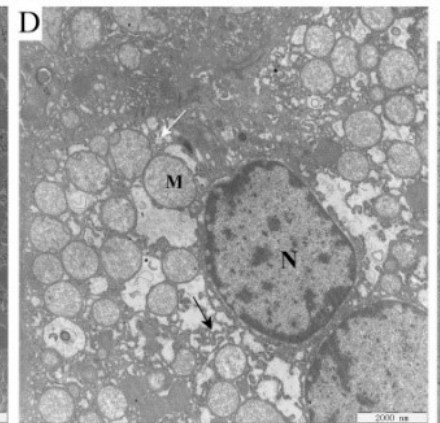

IRI

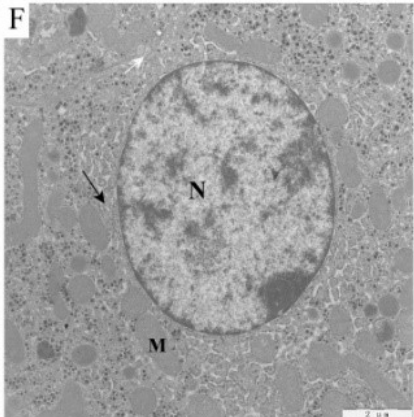

IRI+HRS

$\mathrm{T} 3 \mathrm{~h}$

Fig. 3. Liver cells. A - sham group $(10,000 \times)$; B - sham group $(12,000 \times)$; C - IRI group $(10,000 \times)$; D - IRI group $(12,000 \times) ; \mathrm{E}-\mathrm{IRI}+\mathrm{HRS}$ group $(10,000 \times) ; \mathrm{F}-\mathrm{IRI}+\mathrm{HRS}$ group $(12,000 \times)$; $\mathrm{N}-$ nuclei; $\mathrm{M}$ - mitochondria; black arrows indicate endoplasmic reticulum and white arrows indicate the Golgi apparatus 

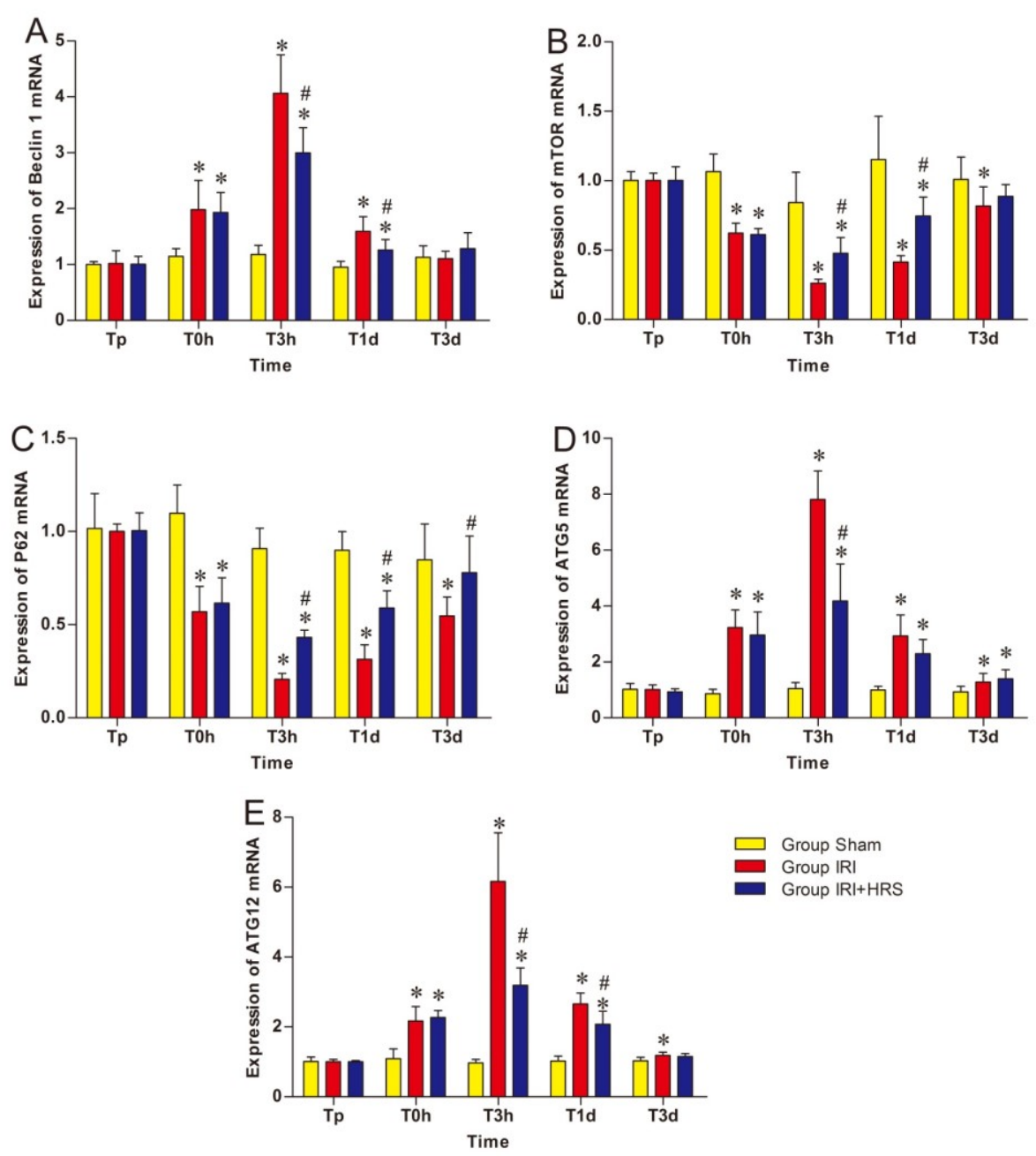

Fig. 4. Expression levels of Beclin1 (A), mTOR (B), p62 (C), ATG5 (D), and ATG12 (E) in the liver. Values are mean \pm standard deviation. *Significant difference from sham group. \# Significant difference of IRI + HRS group from IRI group
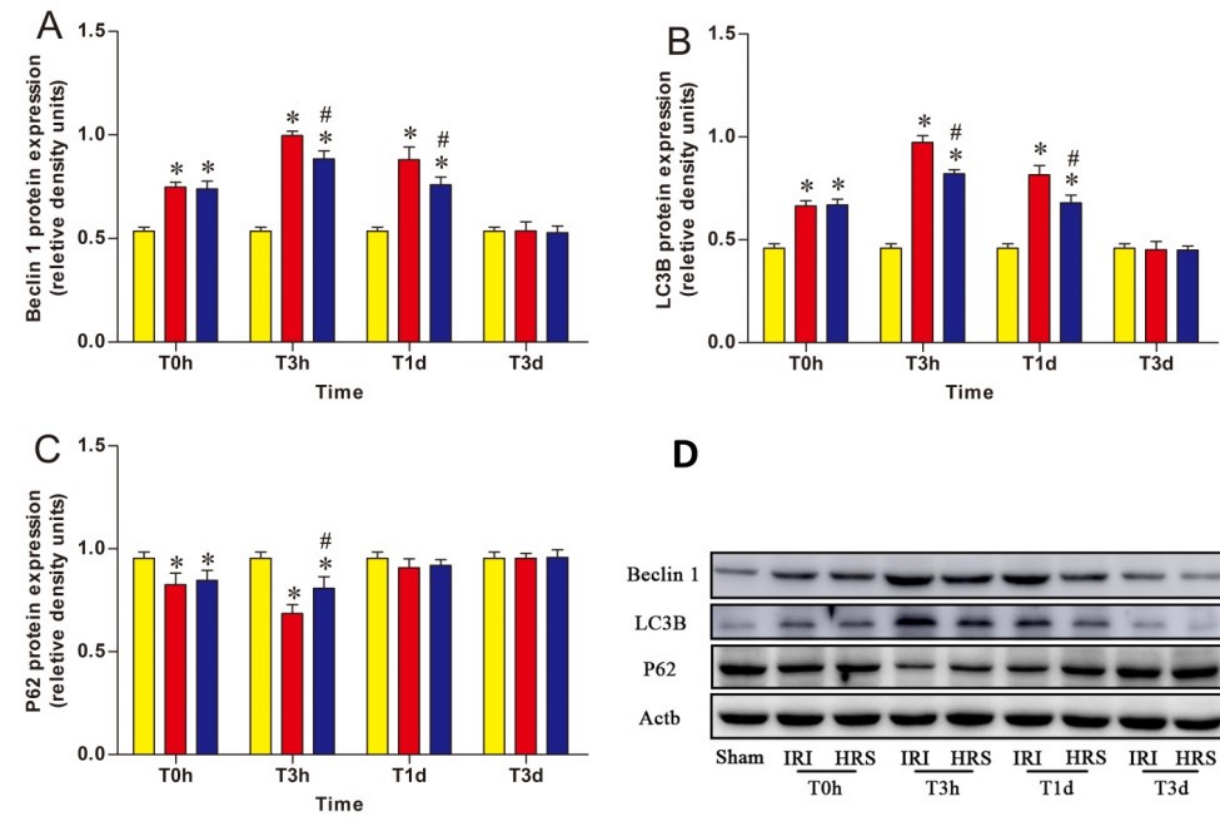

D

$$
\square \text { Group Sham } \square \text { Group IRI } \square \text { Group IRI+HRS }
$$

Fig. 5. Expression levels of Beclin1 (A), LC3B (B), and p62 (C) proteins in the three groups measured by Western blot. (D) Western blot results. Values are mean \pm standard deviation. * Significant difference from sham group. \# Significant difference of IRI + HRS group from IRI group 

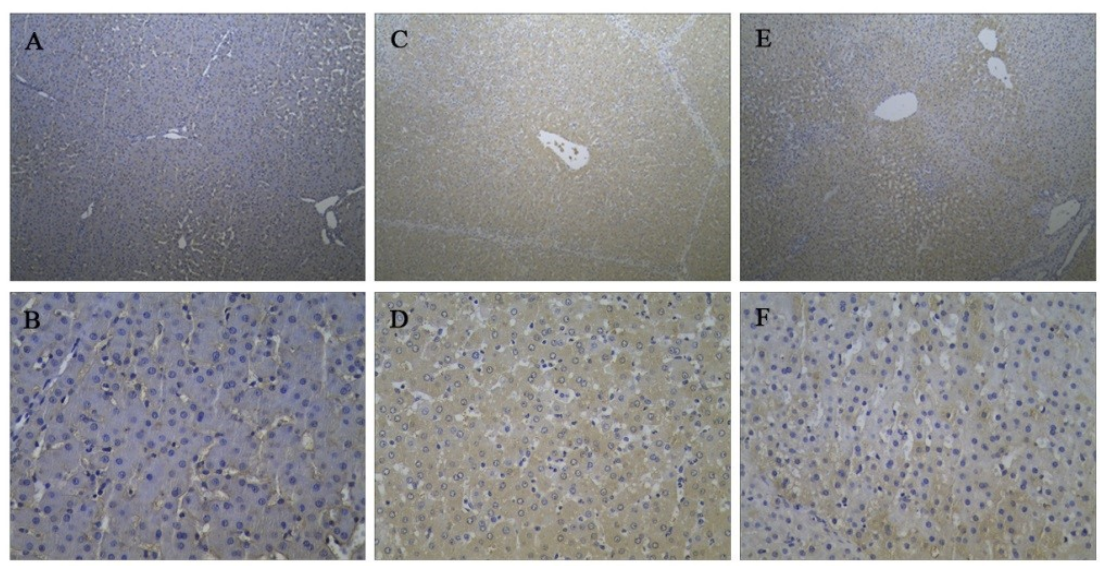

Sham

IRI

IRI+HRS

T3h

Fig. 6. Liver. The results of IHC of LC3B at T3h; A and B - sham group; C and D - IRI group; $\mathrm{E}$ and $\mathrm{F}-\mathrm{IRI}+\mathrm{HRS}$ group. The images $\mathrm{A}, \mathrm{C}$, and $\mathrm{E}$ were recorded at $100 \times$ magnification, and the images $\mathrm{B}, \mathrm{D}$, and $\mathrm{F}$ were recorded at $400 \times$ magnification

\section{Discussion}

Laparoscopic surgery is extensively used on animals nowadays, including for liver surgery (17). Liver ischaemia-reperfusion injury is a common pathophysiological process in liver surgery and transplantation, which can seriously impair liver function. This may also have consequences for multiple other tissues and organs, resulting in a reduced treatment efficacy for patients after surgery (10). The mechanisms of ischaemia-reperfusion injury are still not fully understood. However, ROS and oxidant stress were proven to play a significant role in ischaemiareperfusion injury (23).

During ischaemia, the oxygen content in tissues becomes insufficient, while the restoration of blood flow and the resulting reperfusion leads to an explosive increase in levels of oxygen-free radicals over a short time interval. In addition, endogenous antioxidants such as SOD and CAT, the function of which is to reduce or consume ROS, are able to adequately control the rapid increase in ROS in the liver during ischaemiareperfusion injury. SOD is a metal-containing enzyme which catalyses the disproportion of reactive oxygenfree radicals, and thus eliminates ROS from the body. Serving a complementary function, GSH clears the metabolic byproducts produced by ROS and reduces lipid peroxidation. CAT is known to eliminate the large amount of ROS produced by disproportion of reactions and decomposes other peroxides. In our study, the activity of SOD, GSH, and CAT in liver tissue decreased following liver ischaemia-reperfusion combined resection injury and indicated that the antioxidant capacity within the liver tissue decreased. MDA is the final product of unsaturated lipid peroxidation, and its levels in tissues and circulation can reflect the degree of lipid peroxidation occurrence (4). Numerous studies have demonstrated that a reduction in MDA content correlates to a reduction in ROS-mediated peroxidation damage of tissues (25). In our study, following ischaemia-reperfusion, the level of MDA increased and the examination of liver function showed that the serum ALT, AST, and AKP levels increased. As was corroborated through electron microscopic observations of tissues, hepatocellular submicrostructure damage was inflicted during liver ischaemia-reperfusion combined resection injury. Thus, we believe that the reduction of antioxidant capacity is the mechanism through which ischaemia and reperfusion causes damage to liver tissue.

Furthermore, we demonstrated that the use of HRS during laparoscopic surgery can improve the antioxidative ability of liver tissue, lower its susceptibility to injury, and reduce liver damage, suggesting a hepatoprotective effect of HRS during laparoscopic ischaemia-reperfusion combined resection injury. These findings were also corroborated through liver function tests and electron microscopic observations of tissues. Hydrogen has been wrongly believed by biologists to be a physiologically inert gas for many years. However, in 2007, Japanese researchers observed that inhalation of $2 \%$ hydrogen was able to improve brain oedema and decrease infarct volume of brain focal ischaemia and reperfusion in rats. It was concluded that hydrogen exhibits antioxidant functions (19). Successive studies have found that in the process of ischaemia-reperfusion injury, the free radical/antioxidant system becomes imbalanced, and hydrogen therapy can reduce ischaemia-reperfusion injury caused by such an imbalance (12). As a scavenger of toxic ROS, hydrogen has a selective antioxidant effect, primarily scavenging hydroxyl radicals and nitrite anions, both of which exhibit potent oxidative toxicity. This toxicity can result in peroxidation damage to DNA and lipids, while obliterating various protein functions. In addition, 
hydrogen has been shown to increase antioxidant levels and enzymatic activities of antioxidants (6), thus improving ROS clearance.

Autophagy is an intracellular self-digestion pathway, which functions in the removal of abnormal organelles and proteins, as well as any residual or unnecessary cytoplasmic components through lysosomal digestion (1). Autophagy and the ubiquitinproteasome system (UPS) are the two main pathways for the degradation of specific, short-lived proteins (26). Normally, autophagy plays a very important role in the maintenance of cell survival, cell renewal, recycling of cellular materials, and internal stability. Cursio et al. (3) described that the autophagic cell response to warm and/or cold liver ischaemiareperfusion may delay apoptosis and necrosis and thus can decrease the extent of warm/cold liver ischaemiareperfusion injury. However, over-activation of autophagy can destroy normal proteins and organelles, leading to cell death. In the liver, Gotoh et al. (8) found that inhibited autophagy can reduce the degree of hepatic ischaemia-reperfusion injury after liver transplantation, and Liu et al. (16) found autophagy plays a potential regulatory role in ischaemiareperfusion injury after lung transplantation. Studies have reported that LC3 is a specific protein marker of autophagy and has been shown to increase after ischaemia-reperfusion injury. When autophagy is activated, LC3A is coupled with phosphatidylethanolamine under the action of ubiquitin-like enzyme to produce LC3B (2). Beclin 1 can also be employed as a biomarker of autophagy, as it has been observed to be a regulatory factor upstream of autophagosome formation and contributes to the formation of autophagosomes by binding to phosphoinositide 3-kinase (PI3K) Class III to promote autophagy (29). In addition, Beclin 1 has been shown to regulate most of the phases associated with autophagy, therefore indirectly reflecting the level of autophagy. Another key regulatory factor of autophagy is mTOR. It serves as an effector molecule downstream of the PI3K-Akt signalling pathway, a hub that regulates multiple substances and energy metabolism within the cell, and a negative autophagy regulator (31). The removal of the autophagic substrate must take place through two steps: one is the recognition of the substrate to be degraded by autophagy, and the other is the transport of the substrate to the autophagosomes. These two steps are the key to autologous flow activation taking place or not. As a selective autophagic receptor p62 is the key factor in manipulating these steps. This protein is a ubiquitin-binding protein involved in cell signalling, oxidative stress, and autophagy (14), which combines with ubiquitinated proteins into the autophagosome and eventually fuses with lysosomes to form autophagosomes to be cleared. When p62 is coupled to $\mathrm{LC} 3 \mathrm{~B}$, as a regulator of the composition of autophagosomes, it is degraded in during autophagy as well (20). Formation of the autophagosome involves a ubiquitin-like conjugation system in which ATG12 is covalently bound to ATG5 for subsequent targeting to autophagosome vesicles. The ATG5-ATG12 binding system plays an important role in the binding and recruitment of ATG8 (LC3), which functions as a ubiquitination modification system. This binding system consists of ubiquitinated ATG12, the activating enzyme ATG7, ubiquitinbinding enzyme ATG10, and substrate ATG5.

In our study, the RT-PCR data suggested that the expression of ATG5, ATG12, and Beclin1 increased, while mTOR and p62 decreased following laparoscopic liver ischaemia-reperfusion combined resection injury. Comprehensive data show that this injury led to increased hepatocyte autophagy. In comparison to the IRI group, the IRI + HRS group was observed to have reduced expression of ATG5, ATG12, and Beclin1, whereas the expression of mTOR and p62 increased. The immunohistochemistry data showed that the expression of LC3B increased following laparoscopic ischaemia-reperfusion combined resection injury, while the expression of LC3B decreased in liver samples from animals that received the same surgery and HRS intervention. The IHC and RT-PCR results were also confirmed by Western blot analysis. Western blot also bore out the gene expression patterns observed in the HRS intervention group, namely that Beclin1 and LC3B expression decreased, whereas p62 increased, suggesting an overall decrease in autophagy. These data suggest that laparoscopic liver ischemiareperfusion combined resection injury activates autophagy in the liver, whereas HRS intervention can moderate the autophagy increase brought about by laparoscopic liver ischaemia-reperfusion combined resection injury.

Numerous studies have shown that ROS derived from mitochondria are the major inducer of autophagy under oxidative stress in response to ischaemiareperfusion (5). In the autophagy induction phase, ROS can induce autophagy through the suppression of mTOR through the inhibition of PI3K-Akt-mTOR (22). In the process of autophagosome formation, ROS exert effects resulting in the accumulation of $\mathrm{LC} 3 \mathrm{~B}$, thus increasing autophagy (27). Moreover, ROS can promote the ubiquitination of various cellular proteins to be degraded, which promotes translocation of the degradation product to the autophagosome once it combines with p62 and LC3B, and there it is finally degraded (15). In our study, not only did autophagy increase following laparoscopic liver ischaemiareperfusion combined resection injury, but the animals also experienced severe peroxidation injury in liver tissue. HRS intervention was observed to decrease the level of autophagy in liver tissue and reduce the peroxidation injury as well.

In conclusion, we demonstrated the protective effect of HRS on the liver and its influence on hepatocyte autophagy during laparoscopic liver ischaemia-reperfusion combined resection injury. HRS 
intervention is capable of reducing oxidant stress injury, decreasing the autophagy level in liver tissue, and ultimately protecting the liver during laparoscopic liver ischaemia-reperfusion combined resection injury.

Conflict of Interests Statement: The authors declare that there is no conflict of interests regarding the publication of this article.

Animal Rights Statement: Care of and experimental procedures on the animals were conducted in accordance with the rules for care and use of experimental animals established by the Ministry of Science and Technology of the People's Republic of China (Approval number: 2006-398). The present study was approved by the Northeast Agricultural University Institutional Animal Care and Use Committee (Harbin, China).

Financial Disclosure Statement: This work was supported by the National Natural Science Foundation of China (31772807) and the National Natural Science Foundation of China (31472245).

\section{References}

1. Baehrecke E.H.: Autophagy: dual roles in life and death? Nature Rev Mol Cell Biol 2005, 6, 505-510.

2. Codogno P., Meijer A.J.: Autophagy and signaling: their role in cell survival and cell death. Cell Death Differ 2005, 12, 1509-1518.

3. Cursio R., Colosetti P., Codogno P., Cuervo A M, Shen H.: The role of autophagy in liver diseases: mechanisms and potential therapeutic targets. Biomed Res Int 2015, 2015, 480508.

4. Farre J.C., Subramani S.: Peroxisome turnover by micropexophagy: an autophagy-related process. Trends Cell Biol 2004, 14, 515-523.

5. Filomeni G., De Zio D., Cecconi F.: Oxidative stress and autophagy: the clash between damage and metabolic needs. Cell Death Differ 2015, 22, 377-388.

6. George J.F., Agarwal A.: Hydrogen: another gas with therapeutic potential. Kidney Int 2010, 77, 85-87.

7. Glantzounis G.K., Salacinski H.J., Yang W., Davidson B.R., Seifalian A.M.: The contemporary role of antioxidant therapy in attenuating liver ischemia-reperfusion injury: a review. Liver Transpl 2005, 11, 1031-1047.

8. Gotoh K., Lu Z., Morita M., Shibata M., Koike M., Waguri S., Dono K., Doki Y., Kominami E., Sugioka A., Monden M., Uchiyama Y.: Participation of autophagy in the initiation of graft dysfunction after rat liver transplantation. Autophagy 2009, 5, 351-360.

9. Guo Z., Zhou B., Li W., Sun X., Luo D.: Hydrogen-rich saline protects against ultraviolet $\mathrm{B}$ radiation injury in rats. $\mathrm{J}$ Biomed Res 2012, 26, 365-371.

10. Gurusamy K.S., Kumar Y., Ramamoorthy R., Sharma D., Davidson B.R.: Vascular occlusion for elective liver resections. Cochrane Database Syst Rev 2009, CD007530.

11. Hong J.Y., Lebofsky M., Farhood A., Jaeschke H.: Oxidant stress-induced liver injury in vivo: role of apoptosis, oncotic necrosis, and c-Jun NH2-terminal kinase activation. Am J Physiol-Gastrointest Liver Physiol 2009, 296, 572-581.
12. Huang T.L., Wang W.C., Tu C., Yang Z.Y., Bramwell D., Sun X.J.: Hydrogen-rich saline attenuates ischemia-reperfusion injury in skeletal muscle. J Surg Res 2015, 194, 471-480.

13. Karatzas T., Neri A.A., Baibaki M.E., Dontas I.A.: Rodent models of hepatic ischemia-reperfusion injury: time and percentage-related pathophysiological mechanisms. J Surg Res 2014, 191, 399-412.

14. Kirkin V., Mc Ewan D.G..: A role for ubiquitin in selective autophagy. Mol Cell 2009, 34, 259-269.

15. Lee J., Giordano S., Zhang J.H.: Autophagy, mitochondria, and oxidative stress: cross-talk and redox signalling. Biochem J 2012, 441, 523-540.

16. Liu S., Zhang J., Yu B., Huang L., Dai B., Liu J., Tang J.: The role of autophagy in lung ischemia/reperfusion injury after lung transplantation in rats. Am J Transpl Res 2016, 8, 3593-3602.

17. Mayhew P.D.: Recent advances in soft tissue minimally invasive surgery. J Small Anim Pract 2014, 55, 75-83.

18. Milovancev M., Townsend K.L.: Current concepts in minimally invasive surgery of the abdomen. Vet Clin North Am Small Anim Pract 2015, 45, 507-522.

19. Ohsawa I., Ishikawa M., Takahashi K., Watanabe M., Ohta S.: Hydrogen acts as a therapeutic antioxidant by selectively reducing cytotoxic oxygen radicals. Nature Med 2007, 13, 688-694.

20. Pankiv S., Clausen T.H., Lamark T., Brech A.: p62/SQSTM1 binds directly to Atg8/LC3 to facilitate degradation of ubiquitinated protein aggregates by autophagy. J Biol Chem 2007, 282, 24131-24145.

21. Peralta C., Jimenez-Castro M.B., Gracia-Sancho J.: Hepatic ischemia and reperfusion injury: effects on the liver sinusoidal milieu. J Hepatol 2013, 59, 1094-1106.

22. Portal-Nunez S., Esbrit P., Alcaraz M.J., Largo R.: Oxidative stress, autophagy, epigenetic changes, and regulation by miRNAs as potential therapeutic targets in osteoarthritis. Biochem Pharmacol 2016, 108, 1-10.

23. Rahal A., Kumar A., Singh V., Yadav B., Tiwari R., Chakraborty S., Dhama K.: Oxidative stress, prooxidants, and antioxidants: the interplay. Biomed Res Int 2014, 2, 74-79.

24. Rautou P.E., Mansouri A., Lebrec D., Durand F., Valla D., Moreau R.: Autophagy in liver diseases. J Hepatol 2010, 53, 1123-1134.

25. Rossi R., Dalle-Donne I., Milzani A., Giustarini D.: Oxidized forms of glutathione in peripheral blood as biomarkers of oxidative stress. Clin Chem 2006, 52, 1406-1414.

26. Ryter S.W., Cloonan S.M., Choi A.M.K.: Autophagy: a critical regulator of cellular metabolism and homeostasis. Mol Cells 2013, 36, 7-16.

27. Scherz-Shouval R., Shvets E., Fass E., Shorer H., Gil L., Elazar Z.: Reactive oxygen species are essential for autophagy and specifically regulate the activity of Atg4. Embo J 2007, 26, 1749-1760.

28. Stewart R.K., Dangi A., Gandhi C.R.: A novel mouse model of depletion of stellate cells clarifies their role in ischemia/reperfusion- and endotoxin-induced acute liver injury. J Hepatol 2014, 60, 298-305.

29. Tassa A., Roux M.P., Attaix D., Bechet D.M.: Class III phosphoinositide 3-kinase-Beclin1 complex mediates the amino acid-dependent regulation of autophagy in $\mathrm{C} 2 \mathrm{C} 12$ myotubes. Biochem J 2003, 376, 577-586.

30. Vlahakos D., Arkadopoulos N., Smyrniotis V.: Deferoxamine attenuates lipid peroxidation, blocks interleukin-6 production, ameliorates sepsis inflammatory response syndrome, and confers renoprotection after acute hepatic ischemia in pigs. Artif Organs 2012, 36, 400-408.

31. Xie Z.L., He C.Y., Zou M.H.: AMP-activated protein kinase modulates cardiac autophagy in diabetic cardiomyopathy. Autophagy 2011, 7, 1254-1255. 